\title{
The Optimal q-Homotopy Analysis Method (Oq-HAM)
}

Shaheed N. Huseen, Said R.Grace, Magdy A. El-Tawil

Thi-Qar University, Faculty of Computer Science and Mathematics,

Mathematics Department, Thi-Qar, Iraq

shn_n2002@yahoo.com

Cairo University, Faculty of Engineering,

Engineering Mathematics Department, Giza, Egypt

saidgrace@yahoo.com

Cairo University, Faculty of Engineering,

Engineering Mathematics Department, Giza, Egypt

ABSTRAC

magdyeltawil@yahoo.com

In this paper, an optimal q-homotopy analysis method (Oq-HAM) is proposed. We present some examples to show the reliability and efficiency of the method. It is compared with the one-step optimal homotopy analysis method. The results reveal that the Oq-HAM has more accuracy to determine the convergence-control parameter than the one-step optimal HAM.

Keywords: Optimal q-homotopy analysis method; Convergence-control parameter.

\section{Council for Innovative Research}

Peer Review Research Publishing System

Journal: INTERNATIONAL JOURNAL OF COMPUTERS \& TECHNOLOGY

\author{
Vol 11, No. 8 \\ editor@cirworld.com \\ www.cirworld.com, member.cirworld.com
}




\section{INTRODUCTION}

The search for a better and easy to use tool for the solution of nonlinear equations illuminating the nonlinear phenomena of our life keeps continuing. A variety of methods therefore were proposed to find approximate solutions. Liao [10-14] employed the basic ideas of the homotopy in topology to propose a general analytic method for linear and nonlinear problems, namely homotopy analysis method (HAM). In recent years, this method has been successfully applied to solve many types of nonlinear problems in science and engineering $[6,9,17,20]$. The HAM contains a certain auxiliary parameter $\mathrm{h}$ which provides us with a simple way to adjust and control the convergence region and rate of convergence of the series solution. Moreover, by means of the so-called h-curve, it is easy to determine the valid regions of $h$ to gain a convergent series solution. The use of the convergence-control parameter $h$ is indeed a great progress in the frame of the HAM. It seems that more "artificial" degrees of freedom imply larger possibility to gain better approximations by means of the homotopy analysis method. How to find a proper convergence-control parameter $h$ so as to gain a convergent series solution? A straight-forward way to check the convergence of a homotopy-series solution is to substitute it into original governing equations and boundary/initial conditions and then to check the corresponding squared residual integrated in the whole region.

The $\mathrm{h}$-curves cannot tell us the best convergence-control parameter $\mathrm{h}$, which corresponds to the fastest convergent series. In 2007, Yabushita et al. [19] applied the HAM to solve two coupled nonlinear ODEs. They suggested the so-called "optimization method" to find out the two optimal convergence-control parameters by means of the minimum of the squared residual error of governing equations. In 2008, Akyildiz and Vajravelu [2] gained optimal convergence-control parameter by the minimum of squared residual of governing equation, and found that the corresponding homotopy-series solution converges very quickly, Marinca et al. $[16,17]$ introduced the so-called "homotopy asymptotic method" which is similar to the homotopy analysis method, Niu et.al. [18] proposed a method namely one-step optimal homotopy analysis method, Liao [15] developed in an optimal HAM with only three convergence-control parameters. El-Tawil and Huseen $[3,4]$ proposed a method namely q-homotopy analysis method (q-HAM) which is more general method of homotopy analysis method (HAM), The q-HAM contains an auxiliary parameter $n$ as well as $h$ such that the case of $n=1$ ( $q-H A M$; $\mathrm{n}=1$ ) the standard homotopy analysis method (HAM) can be reached.

In this paper, an optimal q-homotopy analysis method is proposed. This optimal method contains only one convergencecontrol parameter and is computationally rather efficient.

\section{BASIC IDEA OF THE OPTIMAL q-HOMOTOPY ANALYSIS METHOD (Oq-HAM)}

Consider the following differential equation

$$
\mathrm{N}[\mathrm{u}(\mathrm{t})]=0,
$$

where $\mathrm{N}$ is a nonlinear operator, $\mathrm{u}(\mathrm{t})$ is an unknown function.

Let us construct the so-called zero-order deformation equation:

$$
(1-\mathrm{nq}) \mathrm{L}\left[\varnothing(\mathrm{t} ; \mathrm{q})-\mathrm{u}_{0}(\mathrm{t})\right]=\mathrm{F}(\mathrm{n}) \mathrm{qN}[\varnothing(\mathrm{t} ; \mathrm{q})]
$$

Where $\mathrm{F}(\mathrm{n})$ is a nonzero auxiliary function, $\mathrm{n} \geq 1, \mathrm{q} \in\left[0, \frac{1}{\mathrm{n}}\right]$ denotes the so-called embedded parameter, $\mathrm{L}$ is an auxiliary linear operator.Choosing the function $\mathrm{F}(\mathrm{n})$ depends on the given problem. It is obvious that when $\mathrm{q}=0$ and $\mathrm{q}=\frac{1}{\mathrm{n}}$ equation (2) becomes:

$$
\emptyset(\mathrm{t} ; 0)=\mathrm{u}_{0}(\mathrm{t}) \text { and } \varnothing\left(\mathrm{t} ; \frac{1}{\mathrm{n}}\right)=\mathrm{u}(\mathrm{t})
$$

respectively. Thus as $q$ increases from 0 to $\frac{1}{n}$, the solution $\emptyset(t ; q)$ varies from the initial guess $u_{0}(t)$ to the solution $u(t)$.

Having the freedom to choose $u_{0}(t), L, F(n)$ we can assume that all of them can be properly chosen so that the solution $\emptyset(\mathrm{t} ; \mathrm{q})$ of equation (2) exists for $\mathrm{q} \in\left[0, \frac{1}{\mathrm{n}}\right]$. Expanding $\emptyset(\mathrm{t} ; \mathrm{q})$ in Taylor series, one has:

$\emptyset(\mathrm{t} ; \mathrm{q})=\mathrm{u}_{0}(\mathrm{t})+\sum_{\mathrm{m}=1}^{+\infty} \mathrm{u}_{\mathrm{m}}(\mathrm{t}) \mathrm{q}^{\mathrm{m}}$

where

$$
\mathrm{u}_{\mathrm{m}}(\mathrm{t})=\frac{1}{\mathrm{~m} !} \frac{\partial^{\mathrm{m}} \varnothing(\mathrm{t}, \mathrm{q})}{\partial \mathrm{q}^{\mathrm{m}}} \mathrm{q}=0
$$

Assume that $\mathrm{F}(\mathrm{n}), \mathrm{u}_{0}(\mathrm{t})$, $\mathrm{L}$ are so properly chosen such that the series (4) converges at $\mathrm{q}=\frac{1}{\mathrm{n}}$ and

$$
\mathrm{u}(\mathrm{t})=\varnothing\left(\mathrm{t} ; \frac{1}{\mathrm{n}}\right)=\mathrm{u}_{0}(\mathrm{t})+\sum_{\mathrm{m}=1}^{+\infty} \mathrm{u}_{\mathrm{m}}(\mathrm{t})\left(\frac{1}{\mathrm{n}}\right)^{\mathrm{m}}
$$

Defining the vector $\mathrm{u}_{\mathrm{r}}(\mathrm{t})=\left\{\mathrm{u}_{0}(\mathrm{t}), \mathrm{u}_{1}(\mathrm{t}), \mathrm{u}_{2}(\mathrm{t}), \ldots, \mathrm{u}_{\mathrm{r}}(\mathrm{t})\right\}$. Differentiating Equation (2) $\mathrm{m}$ times with respect to $\mathrm{q}$ and then setting $\mathrm{q}=0$ and finally dividing them by $\mathrm{m}$ ! we have the so-called $\mathrm{m}^{\text {th }}$ order deformation equation:

$\mathrm{L}\left[\mathrm{u}_{\mathrm{m}}(\mathrm{t})-\mathrm{k}_{\mathrm{m}} \mathrm{u}_{\mathrm{m}-1}(\mathrm{t})\right]=\mathrm{F}(\mathrm{n}) \mathrm{R}_{\mathrm{m}}\left(\overrightarrow{\mathrm{u}_{\mathrm{m}-1}}(\mathrm{t})\right)$,

where 
$\mathrm{R}_{\mathrm{m}}\left(\mathrm{u}_{\mathrm{m}-1}(\mathrm{t})\right)=\frac{1}{(\mathrm{~m}-1) !} \frac{\partial^{\mathrm{m}-1} \mathrm{~N}[\varnothing(\mathrm{t}, \mathrm{q})]}{\partial \mathrm{q}^{\mathrm{m}-1}} \mathrm{q}=0$

and

$\mathrm{k}_{\mathrm{m}}=\left\{\begin{array}{lr}0 & \mathrm{~m} \leq 1 \\ \mathrm{n} & \text { otherwise }\end{array}\right.$

It should be emphasized that $u_{m}(t)$ for $m \geq 1$ is governed by the linear equation (7) with linear boundary conditions that come from the original problem. Let

$$
\Delta_{\mathrm{m}}=\int_{\Omega}\left(\mathrm{N}\left[\mathrm{U}_{\mathrm{m}}(\mathrm{t})\right]\right)^{2} \mathrm{~d} \Omega,
$$

where $\mathrm{U}_{\mathrm{m}}(\mathrm{t})=\mathrm{u}_{0}(\mathrm{t})+\sum_{\mathrm{k}=1}^{\mathrm{m}} \mathrm{u}_{\mathrm{k}}(\mathrm{t})\left(\frac{1}{\mathrm{n}}\right)^{\mathrm{k}}$ denote the square residual error of the mth-order appro-ximation of the equation (1) integrated in the whole domain $\Omega$, In theory if the square residual error $\Delta_{\mathrm{m}}$ tends to zero, then

$$
\mathrm{U}_{\mathrm{m}}(\mathrm{t})=\sum_{\mathrm{k}=0}^{\mathrm{m}} \mathrm{u}_{\mathrm{k}}(\mathrm{t})\left(\frac{1}{\mathrm{n}}\right)^{\mathrm{k}}
$$

is a series solution of the original equation (1). Besides, at the given order of approximation, the minimum of the squared residual error $\Delta_{\mathrm{m}}$ corresponds to the optimal approximation, hence the optimal value of the convergence-control parameter $\mathrm{n}$ that corresponds to the minimum of $\Delta_{\mathrm{m}}$.

In the one-step optimal HAM, Niu and Wang [18] construct the zeroth-order deformation equation

$$
(1-q) L\left[\varnothing(t ; q)-u_{0}(t)\right]=\left(\sum_{k=1}^{+\infty} c_{k} q^{k}\right) N[\varnothing(t ; q)],
$$

where $L$ is an auxiliary linear operator , $q \in[0,1]$ denotes the so-called embedded parameter $u_{0}(t)$ an initial approximation of $u(t)$ and the series $\sum_{k=1}^{+\infty} c_{k} q^{k}$ converges at $q=1$.

The $m^{\text {th }}$ order deformation equation is:

$$
L\left[u_{m}(t)-x_{m} u_{m-1}(t)\right]=\sum_{k=1}^{m} c_{k} R_{m-k}(t)
$$

Where $R_{S}(t)=\frac{1}{s !} \frac{\partial^{s} N[\varnothing(t, q)]}{\partial q^{s}}$ and $x_{m}=\left\{\begin{array}{cc}0 & m \leq 1 \\ 1 & \text { otherwise }\end{array}\right.$

At the 1 st-order of approximation $\Delta_{1}$ is only dependent upon $c_{1}$, so, the optimal value of $c_{1}$ is obtain by solving the nonlinear algebric equation

$$
\frac{d \Delta_{1}}{d c_{1}}=0
$$

At the 2nd-order, since $c_{1}$ is known, the square residual error $\Delta_{2}$ is only dependent upon $c_{2}$, thus we can gain the optimal value of $c_{2}$ by solving the nonlinear algebraic equation

$$
\frac{d \Delta_{2}}{d c_{2}}=0
$$

and so on.

\section{APPLICATIONS}

Example 3.1: Consider the nonlinear integro-differential Equation [8]

$$
u^{\prime}(t)=-1+\int_{0}^{t} u^{2}(s) d s, t \in[0,1]
$$

With the boundary condition $u(0)=0$

This problem solved by one-step optimal homotopy analysis method [8], so we will solve it by Oq-HAM and compare the results. We choose auxiliary linear operator

$L[\varnothing(t ; q)]=\frac{\partial \varnothing(t ; q)}{\partial t}$, with the property $L[b]=0$, where $b$ is constant.

We define the nonlinear operators

$$
N[\emptyset(t ; q)]=\frac{\partial \emptyset(t ; q)}{\partial t}+1-\int_{0}^{t} \emptyset^{2}(s ; q) d s
$$

We choose the initial approximations $u_{0}(t)=-t$

According to the zeroth-order deformation equation (2) and the $m t h$-order deformation equation (7) with

$$
R_{m-1}(t)=u_{m-1}^{\prime}+\left(1-\frac{k_{m}}{n}\right)-\int_{0}^{t} \sum_{i=0}^{m-1} u_{i}(s) u_{m-1-i}(s) d s
$$

The solution of the $m t h$-order deformation equation (7) for $m \geq 1$ becomes: 


$$
u_{m}(t)=k_{m} u_{m-1}(t)+F(n) \int R_{m-1}(t) d t+b,
$$

where the constant $b$ determined by the initial condition $u_{m}(0)=0$.

Let $F(n)=1-n, n>1$. We now successively obtain:

$u_{1}=-\frac{t^{4}}{12}+\frac{n t^{4}}{12}$

$u_{2}=n\left(-\frac{t^{4}}{12}+\frac{n t^{4}}{12}\right)+\frac{1}{36}(1-n)(-1+n)\left(3 t^{4}+\frac{t^{7}}{7}\right)$

$u_{m}(t, n),(m=3,4, \ldots)$ can be calculated similarly. Then the series solution expression by Oq- HAM can be written in the form:

$$
u(t, n) \cong U_{M}(t, n)=\sum_{k=0}^{M} u_{i}(t, n)\left(\frac{1}{n}\right)^{i}
$$

Equation (12) is a family of approximation solutions to the problem (11) in terms of the convergence-control parameter $n$. It is found that

$$
\begin{aligned}
\Delta_{1}=\frac{47051}{829730304} & +\frac{1}{31912704 n^{4}}+\frac{34951}{829730304 n^{3}}+\frac{171312527}{9680186880 n^{2}}-\frac{7978183}{4148651520 n} \\
\Delta_{2}=\frac{199674191}{1469713454193600}+\frac{297235061}{8398362595392000 n^{8}}-\frac{26307697}{3094133587776000 n^{7}}+\frac{741363805547}{15596959105728000 n^{6}} & 3402153525697 \\
& -\frac{18861903527}{1679672519078400 n^{5}}+\frac{86367892405031}{4367148549603840 n^{4}}-\frac{466356964075843}{109178713740096000 n^{3}}+\frac{3925337612736000 n^{2}}{992536153} \\
& -\frac{96143866153}{8398362595392000 n}
\end{aligned}
$$

$\Delta_{m}(n),(m=3,4, \ldots)$ can be calculated similarly. The residual errors of one-step optimal HAM and Oq-HAM are shown in Table 1. It is obvious that, in this example, the Oq-HAM has more accuracy than the one-step optimal HAM.

Table (1): Comparison between residuals of one-step optimal HAM and optimal q-homotopy analysis method(OqHAM) for problem (11).

\begin{tabular}{ccccc}
\hline Order $\boldsymbol{m}$ & $\boldsymbol{c}_{\boldsymbol{m}}$ & $\Delta_{\boldsymbol{m}}$ one $\boldsymbol{e}-\boldsymbol{s t e p}$ & $\mathbf{n}_{\boldsymbol{m}}$ & $\Delta_{\boldsymbol{m}}(\boldsymbol{O} \boldsymbol{q H A M})$ \\
\hline 1 & -0.945678 & $0.447006 \mathrm{e}-5$ & 18.4087 & $0.447006 \mathrm{e}-5$ \\
2 & $0.571303 \mathrm{e}-3$ & $0.718753 \mathrm{e}-8$ & 30.8848 & $0.128765 \mathrm{e}-8$ \\
3 & $0.260778 \mathrm{e}-4$ & $0.219308 \mathrm{e}-10$ & 37.3405 & $0.175543 \mathrm{e}-12$ \\
4 & $0.147754 \mathrm{e}-5$ & $0.87287 \mathrm{e}-13$ & 39.2368 & $0.419769 \mathrm{e}-16$ \\
\hline
\end{tabular}

Example 3.2: Consider the nonlinear boundary value problem (Troesch's problem) [5]

$$
u^{\prime \prime}(x)=\beta \sinh (\beta u(x))
$$$$
0 \leq x \leq 1
$$

With the boundary conditions $u(0)=0, u(1)=1$. In order to prevent suffering from the strongly nonlinear term $\sinh (\beta u)$, we can use Taylor series expansion of $\sinh (\beta u)$

$$
\sinh (\beta u)=\beta u+\frac{\beta^{3} u^{3}}{6}
$$

Then (13) become

$$
u^{\prime \prime}-\beta\left(\beta u+\frac{\beta^{3} u^{3}}{6}\right)=0
$$

We choose auxiliary linear operator $L[\varnothing(x ; q)]=\frac{\partial^{2} \varnothing(x ; q)}{\partial x^{2}}$ with the property $L\left[c_{0}+c_{1} x\right]=0$, where $c_{0}, c_{1}$ are integral constants. We define a nonlinear operator as

$$
N[\varnothing(x ; q)]=\frac{\partial^{2} \varnothing(x ; q)}{\partial x^{2}}-\beta\left(\beta \emptyset(x ; q)+\frac{[\beta \varnothing(x ; q)]^{3}}{6}\right)
$$

We choose the initial approximations $u_{0}(x)=\alpha x$. According to the zeroth-order deformation equation (2) and the $m t h$ order deformation equation (7) with:

$$
R_{m}\left(\overrightarrow{u_{m-1}}(x)\right)=\frac{d^{2} u_{m-1}}{d x^{2}}-\beta\left(\beta u_{m-1}+\frac{\beta^{3}}{6} \sum_{i=0}^{m-1} u_{m-1-i} \sum_{k=0}^{i} u_{k} u_{i-k}\right)
$$


The solution of the $m t h$-order deformation equation (7) for $m \geq 1$ becomes

$$
u_{m}(x)=k_{m} u_{m-1}(x)+F(n) L^{-1}\left[R_{m}\left(\overrightarrow{u_{m-1}}(x)\right)\right],
$$

with the boundary conditions $u_{m}(0)=0, u_{m}^{\prime}(0)=0$.

Let $F(n)=n-n^{2}, \quad n>1, \beta=1 ; \alpha=0.8453227$. We now successively obtain:

$u_{1}=-0.00503369(1-n) n x^{3}\left(27.9888+x^{2}\right)$

$u_{2}=-0.00503369(1-n) n^{2} x^{3}\left(27.9888+x^{2}\right)+(1-n) n^{2} x^{3}\left(-0.140887+0.00201067 x^{2}+0.00131835 x^{4}\right.$ $\left.+0.0000249786 x^{6}+n\left(0.140887-0.00201067 x^{2}-0.00131835 x^{4}-0.0000249786 x^{6}\right)\right)$

$u_{m},(m=3,4, \ldots)$ can be calculated similarly. Then the series solution expression by Oq- HAM can be written in the form:

$u(x, n) \cong U_{M}(x, n)=\sum_{k=0}^{M} u_{i}(x, n)\left(\frac{1}{n}\right)^{i}$

Equation (15) is a family of approximation solutions to the problem (13) in terms of the convergence-control parameter $n$. It is found that

$\Delta_{1}=0.969484-0.912233 n+0.217667 n^{2}-0.00117102 n^{3}-0.0000684614 n^{4}+4.55424 \times 10^{-7} n^{5}+1.38398 \times 10^{-8} n^{6}$

$\Delta_{2}=3.44617-6.50826 n+4.64661 n^{2}-1.49531 n^{3}+0.179301 n^{4}+0.00940797 n^{5}-0.005118 n^{6}+0.000925837 n^{7}$ $-0.0000451742 n^{8}-9.32413 \times 10^{-6} n^{9}+2.32543 \times 10^{-6} n^{10}-2.48497 \times 10^{-7} n^{11}+1.00811$ $\times 10^{-8} n^{12}$

$\Delta_{m}(n),(m=3,4, \ldots)$ can be calculated similarly.

The $3^{\text {th }}$ order one-step optimal HAM approximation solution is

$$
\begin{aligned}
& U_{3(\text { one-step })}=0.845323 x-3.41525 x^{3}\left(-0.140887-0.00503369 x^{2}\right)+x^{3}\left(0.0282989-0.010797 x^{2}-0.00196242 x^{4}\right. \\
&+0.000208631 x^{6}+0.0000146934 x^{8}+1.85689 \times 10^{-7} x^{10}-0.00209817(-0.140887 \\
&\left.\left.-0.00503369 x^{2}\right)\right)+2 x^{3}\left(-0.182589+0.00260581 x^{2}+0.00170857 x^{4}+0.0000323722 x^{6}\right. \\
&\left.+0.00943151\left(-0.140887-0.00503369 x^{2}\right)\right)
\end{aligned}
$$

The residual errors of one-step optimal HAM and Oq-HAM are shown in Table 2. Table 3 shows the comparison between $U_{3}$ of one-step optimal HAM and $U_{3}$ of Oq-HAM, with the exact solution which indicates that the speed of convergence for Oq-HAM is faster than the one-step optimal HAM.

Table(2):Comparison between residuals of one-step optimal HAM and optimal q-homotopy analysis method(OqHAM) for problem (18) at $\beta=1, \alpha=0.8453227$.

\begin{tabular}{ccccc}
\hline Order $\boldsymbol{m}$ & $\boldsymbol{c}_{\boldsymbol{m}}$ & $\Delta_{\boldsymbol{m}} \boldsymbol{o n e}-\boldsymbol{s t e p}$ & $\boldsymbol{n}_{\boldsymbol{m}}$ & $\Delta_{\boldsymbol{m}}(\boldsymbol{O q H A M})$ \\
\hline 1 & -1.13842 & $0.124056 \mathrm{e}-2$ & 2.13842 & $0.124056 \mathrm{e}-2$ \\
2 & $0.943151 \mathrm{e}-2$ & $0.310805 \mathrm{e}-4$ & 2.04981 & $0.170894 \mathrm{e}-5$ \\
3 & $-0.209817 \mathrm{e}-2$ & $0.18712 \mathrm{e}-5$ & 2.04865 & $0.978006 \mathrm{e}-8$ \\
\hline
\end{tabular}

Table (3): Comparison between $U_{3}$ of one-step optimal HAM , $U_{3}$ of Oq-HAM and the exact solution of problem (18) at $\beta=1, \alpha=0.8453227$.

\begin{tabular}{cccc}
\hline $\boldsymbol{x}$ & $\boldsymbol{U}_{\mathbf{3}}$ (One-step) & $\boldsymbol{U}_{\mathbf{3}}$ (Oq-HAM) & Exact Solution \\
\hline 0.1 & 0.0846743 & 0.0846733 & 0.0817969966 \\
0.2 & 0.170204 & 0.170196 & 0.1645308709 \\
0.3 & 0.257457 & 0.257431 & 0.2491673608 \\
0.4 & 0.347333 & 0.347273 & 0.3367322092 \\
0.5 & 0.440774 & 0.440662 & 0.428347161 \\
0.6 & 0.538789 & 0.538608 & 0.5252740296 \\
0.7 & 0.642473 & 0.64221 & 0.6289711434 \\
0.8 & 0.753042 & 0.752687 & 0.7411683782 \\
0.9 & 0.871863 & 0.871418 & 0.8639700206 \\
1.0 & 1.00051 & 0.999987 & 1.00000000020 \\
\hline
\end{tabular}


Example 3.3. Consider the following system of Volterra integro-differential equations [8]

$$
\begin{array}{ll}
u_{1}^{\prime}=1+t+t^{2}-u_{2}(t)-\int_{0}^{t}\left(u_{1}(s)+u_{2}(s)\right) d s, & u_{1}(0)=1, \\
u_{2}^{\prime}=-1-t+u_{1}(t)-\int_{0}^{t}\left(u_{1}(s)-u_{2}(s)\right) d s, & u_{2}(0)=-1,
\end{array}
$$

With exact solutions $u_{1}(t)=t+\exp (t)$ and $u_{2}(t)=t-\exp (t)$.

This problem solved by one-step optimal homotopy analysis [8], so we will solve it by Oq-HAM and compare the results.

We choose auxiliary linear operator $L\left[\emptyset_{i}(t ; q)\right]=\frac{\partial \emptyset_{i}(t ; q)}{\partial t}$ with the property $L\left[b_{i}\right]=0$, where $b_{i}$ are constants. We define the nonlinear operators

$$
\begin{aligned}
& N_{1}\left[\emptyset_{1}(t ; q)\right]=\frac{\partial \emptyset_{1}(t ; q)}{\partial t}-\left(1+t+t^{2}\right)+\emptyset_{2}(t ; q)+\int_{0}^{t}\left(\emptyset_{1}(s ; q)+\emptyset_{2}(s ; q)\right) d s \\
& N_{2}\left[\emptyset_{2}(t ; q)\right]=\frac{\partial \emptyset_{2}(t ; q)}{\partial t}+(1+t)-\emptyset_{1}(t ; q)+\int_{0}^{t}\left(\emptyset_{1}(s ; q)-\emptyset_{2}(s ; q)\right) d s
\end{aligned}
$$

We choose the initial approximations $u_{1,0}(t)=\exp (t)$ and $u_{2,0}(t)=-\exp (t)$. According to the zeroth-order deformation equation (2) and the $m t h$-order deformation equation (7) with

$$
\begin{gathered}
R_{1, m-1}(t)=u_{1, m-1}^{\prime}-\left(1-\frac{k_{m}}{n}\right)\left(1+t+t^{2}\right)+u_{2, m-1}+\int_{0}^{t}\left(u_{1, m-1}+u_{2, m-1}\right) d s \\
R_{2, m-1}(t)=u_{2, m-1}^{\prime}-\left(1-\frac{k_{m}}{n}\right)(1+t)-u_{1, m-1}+\int_{0}^{t}\left(u_{1, m-1}-u_{2, m-1}\right) d s
\end{gathered}
$$

The solution of the $m t h$-order deformation equation (7) for $m \geq 1$ becomes

$$
u_{i, m}(t)=k_{m} u_{i, m-1}(t)+F(n) \int R_{i, m-1}(t) d t+b_{i}, i=1,2,
$$

where the constants $b_{i}$ determined by the initial conditions $u_{1, m}(0)=0, u_{2, m}(0)=0$.

Let $F(n)=-\frac{15}{\sqrt[3]{n}}$. We now successively obtain:

$u_{1,1}=-\frac{15\left(-t-\frac{t^{2}}{2}-\frac{t^{3}}{3}\right)}{n^{1 / 3}}, u_{2,1}=-\frac{15\left(-t+\frac{t^{2}}{2}\right)}{n^{1 / 3}}$

$u_{1,2}=-15 n^{2 / 3}\left(-t-\frac{t^{2}}{2}-\frac{t^{3}}{3}\right)-\frac{75\left(12 t+12 t^{2}+6 t^{3}+\frac{t^{5}}{5}\right)}{4 n^{2 / 3}}, u_{2,2}=-15 n^{2 / 3}\left(-t+\frac{t^{2}}{2}\right)-\frac{75\left(12 t-12 t^{2}-2 t^{3}+\frac{t^{5}}{5}\right)}{4 n^{2 / 3}}$

$u_{i, m}(t, n),(i=1,2),(m=3,4, \ldots)$ can be calculated similarly. Then the series solution expression by Oq- HAM can be written in the form

$u_{i}(t, n) \cong U_{i, M}(t, n)=\sum_{k=0}^{M} u_{i, k}(t, n)\left(\frac{1}{n}\right)^{k}$

Equation (17) is a family of approximation solutions to the problem (16) in terms of the convergence parameters $n$. It is found that

$\Delta_{1,1}=\frac{37}{10}+\frac{1680295}{1008 n^{8 / 3}}-\frac{3287}{21 n^{4 / 3}}, \Delta_{2,1}=\frac{1}{3}+\frac{117175}{1008 n^{8 / 3}}-\frac{23}{6 n^{4 / 3}}$

$\Delta_{1,2}=\frac{37}{10}+\frac{8652856525}{16016 n^{16 / 3}}-\frac{221776615}{1848 n^{4}}+\frac{2389025}{252 n^{8 / 3}}-\frac{6574}{21 n^{4 / 3}}, \Delta_{2,2}=\frac{1}{3}+\frac{20294675}{176 n^{16 / 3}}-\frac{742645}{56 n^{4}}+\frac{104845}{252 n^{8 / 3}}-\frac{23}{3 n^{4 / 3}}$

$\Delta_{i, m}(n),(i=1,2),(m=3,4, \ldots)$ can be calculated similarly.

At the 4th-order of approximation, in order to determine the optimal value of $n$, each of the equations in (18) is solved separately. So, the obtained values and corresponding square residual errors are $n=8.5685, \Delta_{1,4}=0.522938 \times 10^{-4}$, $\Delta_{2,4}=0.624163 \times 10^{-3}$ for the first equation and $n=7.8094, \Delta_{1,4}=0.406663 \times 10^{-3}, \Delta_{2,4}=0.782 \times 10^{-6}$ for the second one. So, the minimum of the $\Delta_{1,4}$ and $\Delta_{2,4}$ is correspond to the optimal value of $n$. Thus, $n=7.80942$ is chosen. This procedure leads to the best approximate solution of the system.

The $4^{\text {th }}$ order one-step optimal HAM approximation solutions are

$$
\begin{aligned}
& U_{1,4(\text { one-step })}=e^{t}+0.907434 t+0.082059 t^{2}+0.0958009 t^{3}-0.0104494 t^{4}-0.0103389 t^{5}+0.0006966315 t^{6} \\
& +0.000394245 t^{7}-0.00000271121 t^{9} \\
& U_{2,4(\text { one-step })}=-e^{t}+0.907434 t-0.082059 t^{2}+0.04109446 t^{3}+0.0104494 t^{4}-0.00615912 t^{5}-0.0006966315 t^{6}+ \\
& 0.000195207 t^{7}-0.00000271121 t^{9} \text {. }
\end{aligned}
$$

The residual errors of one-step optimal HAM and Oq-HAM are shown in Table 4. The comparison of $U_{1,4}, U_{2,4}$ given by one-step optimal HAM and Oq-HAM with the exact solutions $u_{1}$ and $u_{2}$ is shown in Table 5 . The absolute errors of the $4^{\text {th }}$ 
order solutions Oq-HAM approximate compared with $4^{\text {th }}$ order solutions one-step optimal HAM are calculated by the formula

$$
\text { Absolute Error }=\left|u_{\text {exact }}-u_{\text {apprax }}\right|
$$

Figures (1) and (2) show that the series solutions obtained by Oq-HAM converge faster than one-step optimal HAM.

Table(4). comparison between residuals of one-step optimal HAM and optimal q-homotopy analysis method(OqHAM) for problem (16).

\begin{tabular}{ccccccc}
\hline $\boldsymbol{m}$ & $\boldsymbol{c}_{\boldsymbol{m}}$ & $\Delta_{\mathbf{1}, \boldsymbol{m}}$ One-step & $\Delta_{\mathbf{2}, \boldsymbol{m}}$ One-step & $\boldsymbol{n}_{\boldsymbol{m}}$ & $\Delta_{\mathbf{1}, \boldsymbol{m}}($ OqHAM $)$ & $\Delta_{\mathbf{2}, \boldsymbol{m}}($ OqHAM $)$ \\
\hline 1 & -0.7042 & $0.2569 \mathrm{e}-1$ & 0.4096 & 9.9147 & $0.256897 \mathrm{e}-1$ & 0.40959 \\
2 & 0.10920 & $0.1663 \mathrm{e}-1$ & $0.5987 \mathrm{e}-1$ & 8.1248 & 0.119505 & $0.226699 \mathrm{e}-3$ \\
3 & $0.3667 \mathrm{e}-1$ & $0.5691 \mathrm{e}-2$ & $0.2925 \mathrm{e}-1$ & 8.0229 & $0.104745 \mathrm{e}-3$ & $0.516831 \mathrm{e}-2$ \\
4 & $0.2266 \mathrm{e}-1$ & $0.3091 \mathrm{e}-2$ & $0.1343 \mathrm{e}-1$ & 7.8094 & $0.406663 \mathrm{e}-3$ & $0.782 \mathrm{e}-6$ \\
\hline
\end{tabular}

Table(5): Comparison of $U_{1,4}, U_{2,4}$ given by one-step optimal HAM and Oq-HAM with the exact solutions $\mathbf{u}_{1}, \mathbf{u}_{2}$ for problem (16).

\begin{tabular}{ccccccc}
\hline $\mathbf{t}$ & $\mathbf{U}_{\mathbf{1}, \mathbf{4}}$ One-step & $\mathbf{U}_{\mathbf{2}, \mathbf{4}}$ One-step & $\mathbf{U}_{\mathbf{1 , 4}}$ Oq-HAM & $\mathbf{U}_{\mathbf{2 , 4}}$ Oq-HAM & $\mathbf{u}_{\mathbf{1}}$ exact & $\mathbf{u}_{\mathbf{2}}$ exact \\
\hline 0 & 1 & -1 & 1 & -1 & 1 & -1 \\
0.2 & 1.40692 & -1.04285 & 1.4214 & -1.02139 & 1.4214 & -1.0214 \\
0.4 & 1.87369 & -1.13915 & 1.89168 & -1.09173 & 1.89182 & -1.09182 \\
0.6 & 2.4147 & -1.29747 & 2.42104 & -1.22188 & 2.42212 & -1.22212 \\
0.8 & 3.04565 & -1.52895 & 3.0213 & -1.42549 & 3.02554 & -1.42554 \\
1 & 3.78388 & -1.84803 & 3.70627 & -1.71962 & 3.718281 & -1.718281 \\
\hline
\end{tabular}

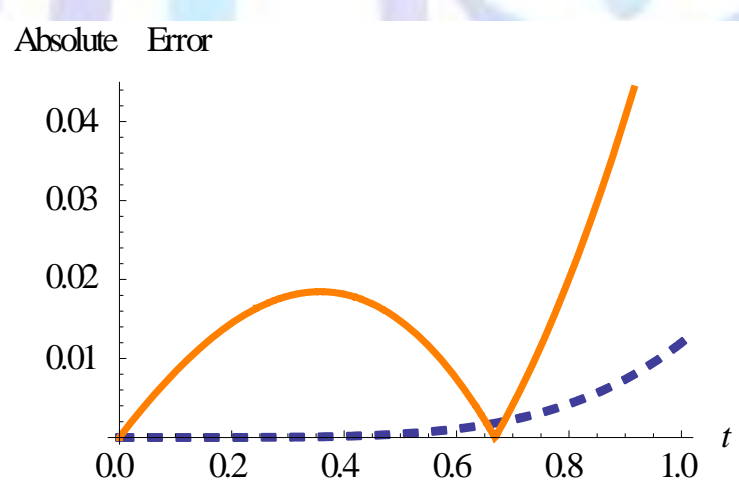

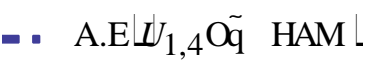

$$
\begin{aligned}
& \text { - A.EL } \perp_{1,4} \text { oné step L }
\end{aligned}
$$

Figure (1): The absolute error of $U_{1,4}$ of one-step optimal HAM and $U_{1,4}$ Oq-HAM for problem (16) . Absolute Error

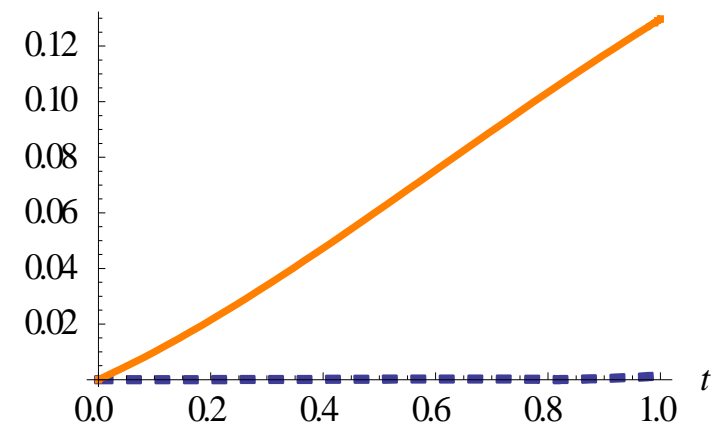

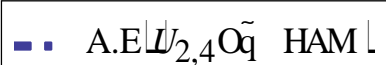

- A.EL $\nu_{2,4}$ onẽ step L

Figure (2): The absolute error of $U_{2,4}$ of one-step optimal HAM and $U_{2,4}$ Oq-HAM for problem (16) . 


\section{CONCLUSION}

In this article, the optimal q-homotopy analysis method (Oq-HAM) is compared with the one-step optimal homotopy analysis method. In order to illustrate the differences between these methods, we solved different types of problems. The results compared show that the Oq-HAM gives better approximations than the one-step optimal HAM.

\section{REFERENCES}

[1] Abbasbandy S., "Soliton solutions for the 5th-order KdV equation with the homotopy analysis method, Nonlinear Dynamics, vol. 51, no. 1-2, pp. 83-87, 2008.

[2] Akyildiz F.T., Vajravelu, K. Magneto hydrodynamic flow of a viscoelastic fluid. Phys. Lett. A. $372,3380-3384$ (2008).

[3] El-Tawil M. A. and Huseen S. N., The q-Homotopy Analysis Method (q-HAM), International Journal of Applied mathematics and mechanics, 8 (15): 51-75, 2012.

[4] El-Tawil M. A. and Shaheed N. Huseen, On Convergence of The q-Homotopy Analysis Method, Int. J. Contemp. Math. Sciences, Vol. 8, 2013, no. 10, $481-497$.

[5] Hany Nasr Hassan Mohammed, The application of homotopy analysis method in solving initial and boundary value problems., Ph.D. thesis ,Faculty of engineering, Cairo University, 2010.

[6] Hayat T., Khan M. and Ayub M., "On non-linear flows with slip boundary condition," Zeitschrift f "ur Angewandte Mathematik und Physik, vol. 56, no. 6, pp. 1012-1029, 2005.

[7] Hayat T., Javed T., Sajid M., Analytic solution for rotating aow and heat transfer analysis of a third-grade auid, Acta Mech. 191 (2007) 219-29.

[8] Jafar S.Nadjafi and Hossein S.Jafari , Comparison of Liao's optimal HAM and Niu's one-step optimal HAM for solving integro-differential equations, Journal of Applied Mathematics and Bioinformatics, vol.1, no.2, 2011, 8598.

[9] Jafari H., Saeidy M. and Firoozjaee M. A., "The homotopy analysis method for solving higher dimensional initial boundary value problems of variable coefficients, Numerical Methods for Partial Differential Equations, vol. 26 , no. 5, pp. 1021-1032, 2010.

[10] Liao S. J., The proposed homotopy analysis technique for the solution of nonlinear problems, Ph.D. thesis, Shanghai Jiao Tong University, 1992.

[11] Liao S. J. , Beyond Perturbation: Introduction to the Homotopy Analysis Method, Chapman \& Hall/CRC, Boca Raton, Fla, USA, 2003.

[12] Liao S. J., "On the homotopy analysis method for nonlinear problems," Applied Mathematics and Computation, vol. 147, no. 2, pp. 499-513, 2004.

[13] Liao S. J. , "Comparison between the homotopy analysis method and homotopy perturbation method," Applied Mathematics and Computation, vol. 169, no. 2, pp. 1186-1194, 2005.

[14] Liao S. J. , "Homotopy analysis method: a new analytical technique for nonlinear problems," Communications in Nonlinear Science and Numerical Simulation, vol. 2, no. 2, pp. 95-100, 1997.

[15] Liao S. J., An optimal homotopy-analysis approach for strongly nonlinear differential equations. Commun. Nonlinear Sci. Numer. Simulat. 15, 2003 - 2016 (2010).

[16] Marinca V., Herişanu N., An optimal homotopy asymptotic method applied to the steady flow of a fourth-grade fluid past a porous plate,Applied Mathematics Letters, 22, (2009), $245-251$.

[17] Marinca V., Herişanu N.and Nemes I., Optimal homotopy asymptotic method with application to thin film flow, Central European Journal of Physics, 6(3), (2008), 648 - 653.

[18] Niu Z. and Wang C., A one-step optimal homotopy analysis method for nonlinear differential equations, Communications in Nonlinear Science and Numerical Simulation, 15, (2010), 2026 - 2036.

[19] Yabushita K., Yamashita M. and Tsuboi K.: An analytic solution of projectile motion with the quadratic resistance law using the homotopy analysis method. J. Phys. A - Math. Theor. 40,8403 - 8416 (2007).

[20] Zhu S. P., "An exact and explicit solution for the valuation of American put options," Quantitative Finance, vol. 6, no. 3, pp. 229-242, 2006. 